The Astrophysical Journal, 485:383-388, 1997 August 10

(C) 1997. The American Astronomical Society. All rights reserved. Printed in U.S.A.

\title{
THE POWER OUTPUT OF SPINE AND FAN MAGNETIC RECONNECTION SOLUTIONS
}

\author{
I. J. D. Craig, R. B. Fabling, and P. G. Watson \\ University of Waikato, Hamilton, New Zealand \\ Received 1996 November 6; accepted 1997 March 4
}

\begin{abstract}
The ability of three-dimensional magnetic "spine" and "fan" reconnection solutions to provide flarelike energy release is discussed. It is pointed out, on the basis of exact analytic solutions, that fast dissipation is possible only if the hydromagnetic pressure in the reconnection region becomes unbounded in the limit of small plasma resistivities. The implication is that some "saturation" of the power output is inevitable for realistic coronal plasmas. Estimates of the saturated power, based on limiting the flux pileup in the field, suggest that the geometry of the spine reconnection mechanism precludes significant flare energy release. However, the current sheet structures involved in fan reconnection seem able to release sufficient magnetic energy fast enough to account for modest flares, even under the conservative assumption of classical plasma resistivities.
\end{abstract}

Subject headings: MHD — plasmas

\section{INTRODUCTION}

It is widely believed that magnetic reconnection is the mechanism underlying the spectacular energy release of the solar flare (Forbes \& Priest 1987; Biskamp 1994). Yet for reconnection to be effective in a plasma of high conductivity, localized regions of strong current density must develop, involving near-singularities in the magnetic field. Models of magnetic merging displaying these characteristics have been constructed, but it seems doubtful whether any resistive mechanism yet developed can liberate sufficient magnetic energy fast enough to explain the flare.

A feature common to all magnetic reconnection solutions is the presence of intense, high-current regions in the vicinity of the magnetic neutral point. This occurs in both dynamic, planar simulations (Biskamp \& Welter 1980; DeLuca \& Craig 1992; Rickard \& Craig 1993; Rickard \& Priest 1994) and in analytic, steady state solutions in two and three dimensions (Craig \& Henton 1995; Craig \& Fabling 1996). The evidence suggests that the ohmic dissipation rate can remain "fast," with reductions in the plasma resistivity $(\eta)$ only if strong "flux pileup" can be sustained by the flow.

Consider, for instance, a typical reconnection simulation in planar geometry (e.g., Hassam 1992; Ofman, Morrison, \& Steinolfson 1993). There is a ground-state configuration - usually a magnetic $X$-point —onto which some global magnetic disturbance is superposed. As time evolves, the disturbance becomes concentrated into a nearsingular current sheet. The length of the sheet is determined externally (by the amplitude of the initial disturbance within limits set by the global geometry), but the width is controlled by the plasma resistivity, $\eta$ (Craig \& Watson 1992; Craig 1994; McClymont \& Craig 1996). Fast reconnection in plasmas of finite pressure occurs provided that the field strength at the onset of the current layer can continue to increase with reductions in $\eta$. However, the merging stalls when the hydromagnetic back pressure in the sheet overcomes the "external" Lorentz forces driving the flow (Biskamp \& Welter 1980; Craig, Henton, \& Rickard 1993; Biskamp 1994; McClymont \& Craig 1996).

These findings seem robust to the details of the reconnection model. Although it is sometimes stated that arbitrary reconnection rates can be achieved in steady state merging solutions, the truth is that all exact models imply some "saturation" of the dissipation rate. This can be demonstrated in "closed" or periodic $X$-point geometries by performing a magnetofrictional relaxation experiment at zero resistivity to determine the amplitude of the saturated field (Rickard \& Craig 1993; Craig 1994). The recent threedimensional "spine" and "fan" reconnection solutions in incompressible "open" plasmas (Craig \& Fabling 1996) also imply saturation. In this case fast reconnection requires that massive hydromagnetic pressures $\left(\sim \eta^{-1 / 2}\right.$ or greater $)$ be available to drive the flows that localize the current. Since there is an upper limit to the pressure available in the solar atmosphere, the flux pileup must eventually saturate, stalling the reconnection.

The likelihood of saturation does not mean, however, that the energetic output of the merging is negligible. As Watson \& Craig (1997a, 1997b) point out, the power output from fan reconnection solutions can be quite considerable, even under the assumption of classical collisional resistivities. In fact, since a flare probably involves significant enhancements of the classical resistivity by factors of $10^{5}$ or more (Parker 1979), the power estimates of Watson $\&$ Craig could be significantly underestimated.

The purpose of the present paper is to assess how the energy outputs from spine and fan reconnection measure up against the extreme requirements of the flare. We consider mainly classical, uniform resistivities, but assume that the hydromagnetic pressures driving the merging can never exceed the level set by photospheric sunspot fields (typically a few thousand gauss) at the base of the solar atmosphere. These broad assumptions are all that is necessary to estimate the saturated output power of the reconnection solutions.

In $\S 2$ we compare the predictions of recent analytic, three-dimensional reconnection solutions against the energetic requirements of the flare. A discussion of the saturated power outputs is given in $\S 3$, where it is pointed out that the spine reconnection output is insufficient for the flare. A simple geometrical argument shows that fan reconnection solutions provide energy release scalings which are near optimum. Our main conclusions are summarized in $\S 4$. 


\section{SPINE AND FAN RECONNECTION SOLUTIONS}

\subsection{MHD Equations}

We assume that the plasma is governed by the steady state momentum and induction equations for an incompressible, resistive plasma. The geometry is open in the sense that material can enter and leave the reconnection region. The system can be written in the nondimensional form

$$
\begin{gathered}
(v \cdot \nabla) \omega-(\omega \cdot \nabla) v=(B \cdot \nabla) J-(J \cdot \nabla) B \\
(v \cdot \nabla) B-(B \cdot \nabla) v=\eta \nabla^{2} B,
\end{gathered}
$$

where the magnetic and velocity fields satisfy $\nabla \cdot \boldsymbol{B}=$ $\nabla \cdot v=0$, and the current density and fluid vorticity are given by

$$
J=\nabla \times B, \quad \omega=\nabla \times v .
$$

The system is conservative apart from the resistive losses of the plasma. The dimensionless coronal resistivity $\eta$ has the magnitude of an inverse Lundquist number. Lundquist numbers from $10^{-14}$ to $10^{-10}$ have been quoted in the literature, and we shall assume a logarithmic mean $\eta=$ $\eta_{c}=10^{-12}$ in what follows. The smallness of the resistivity implies that the ohmic dissipation rate,

$$
W_{\eta}=\eta \int J^{2} d V \equiv \eta\left\langle J^{2}\right\rangle,
$$

can be significant only in the presence of steep gradients in the magnetic field. A central aim of reconnection theory is to demonstrate solutions that-for well-defined $\boldsymbol{B}$ and $\boldsymbol{v}$ fields on the inflow boundaries - can produce strong localized currents in the vicinity of the magnetic neutral point. The solution is "fast" if $W_{\eta}$ scales independently of any positive power of the plasma resistivity. In practice this requires $\left\langle J^{2}\right\rangle$ to grow faster than $1 / \eta$ as $\eta \rightarrow 0$.

\subsection{Energetic Requirements of the Flare}

It is generally accepted that only fast dissipation mechanisms can explain the flare. Suppose we consider a coronal plasma of field strength $B_{c}=100 \mathrm{G}$ occupying the volume $V_{c}=L_{c}^{3}$, where $L_{c}=10^{9.5} \mathrm{~cm}$. The typical Alfvén speed $v_{\mathrm{A}}=10^{8.5} \mathrm{~cm} \mathrm{~s}^{-1}$, corresponding to a plasma of number density $10^{10} \mathrm{~cm}^{-3}$, leads to an Alfvén timescale of $\tau_{\mathrm{A}}=$ $L_{c} / v_{\mathrm{A}}=10 \mathrm{~s}$. The flare release time, typically $10^{2} \mathrm{~s}$, corresponds to several coronal Alfvén times.

However, although the global magnetic energy of the plasma is $10^{31}$ ergs, only a small fraction is required to power a modest flare. A global reduction of just $1 \mathrm{G}$ yields an output of $10^{29.5}$ ergs, which is directly comparable to the thermal content of a soft X-ray-emitting flare plasma of $10^{7}$ $\mathrm{K}$. Yet the smallness of the resistivity makes it is difficult for any resistive mechanism to achieve the required power output of $10^{27}-10^{28} \mathrm{ergs} \mathrm{s}^{-1}$.

Suppose we calibrate $W_{\eta}$ in units of the global magnetic energy $B_{c}^{2} / 8 \pi$ divided by the Alfvén time $\tau_{\mathrm{A}}$. Then $W_{\eta}$ must be of order $10^{-3}$ to meet the rate of $10^{27} \mathrm{ergs} \mathrm{s}^{-1}$. We see that in the coronal limit $\eta=\eta_{c} \simeq 10^{-12}$, the dimensionless current density has to be enormous, $\left\langle J^{2}\right\rangle \simeq 10^{9}$, to achieve an appreciable flare output.

\subsection{Spine and Fan Reconnection Models}

As Craig \& Fabling (1996) have pointed out, any reconection solution is likely to be related to the eigenstructure of three-dimensional magnetic nulls (see Lau \& Finn 1990; Priest \& Titov 1996). Consider the three-dimensional $X$-point given by

$$
\boldsymbol{P}(\boldsymbol{x})=\alpha[-x \hat{\boldsymbol{x}}+\kappa y \hat{\boldsymbol{y}}+(1-\kappa) z \hat{\boldsymbol{z}}] .
$$

Here $\kappa$ defines the isotropy of the field, $\alpha$ the intensity, and all variables are measured in the coronal units introduced in $\S 2.2$. By taking $0<\kappa<1$ and $\alpha>0$, the $x$-axis always defines a separatrix field line into the neutral point; outgoing separatrix lines are restricted to the plane $x=0$ and emanate radially from the null - as illustrated schematically in Figure 1. In the nomenclature of Priest \& Titov (1996), the separatrices comprise the spine curve, namely, the $x$-axis, and the fan plane $x=0$.

Reconnection is driven by superposing disturbances onto the $X$-point. Craig \& Fabling (1996) show that the form of the reconnection depends on which of the separatrices is distorted by the disturbance field $Q(x)$. The magnetic and velocity fields are given by the general superposition

$$
\boldsymbol{B}(\boldsymbol{x})=\lambda \boldsymbol{P}+\boldsymbol{Q}, \quad \boldsymbol{v}(\boldsymbol{x})=\boldsymbol{P}+\lambda \boldsymbol{Q},
$$

where $\lambda$ is a constant and $\boldsymbol{P}$ is the $X$-point field (eq. [2.5]).

In fan current reconnection we have that

$$
\boldsymbol{Q}=\boldsymbol{Q}(x)=Y(x) \hat{\boldsymbol{y}}+Z(x) \hat{\boldsymbol{x}},
$$

and so only distortions of the spine occur. As shown schematically in Figure 2, this leads to a classical current sheet in the fan plane $x=0$. Flux is piled up at the onset of a narrow resistive layer overlying the neutral point. By taking $\lambda=0$, the solution degenerates to pure magnetic annihilation involving straight field lines.

Figures 3 illustrates a second possibility, namely, that quasi-cylindrical, spine-aligned currents are driven by disturbances in the fan. In this case the disturbance field is two dimensional,

$$
Q(y, z)=X(y, z) \hat{\boldsymbol{x}},
$$

and the reconnection solution displays counterrotating current tubes aligned parallel to the axis of the spine. The

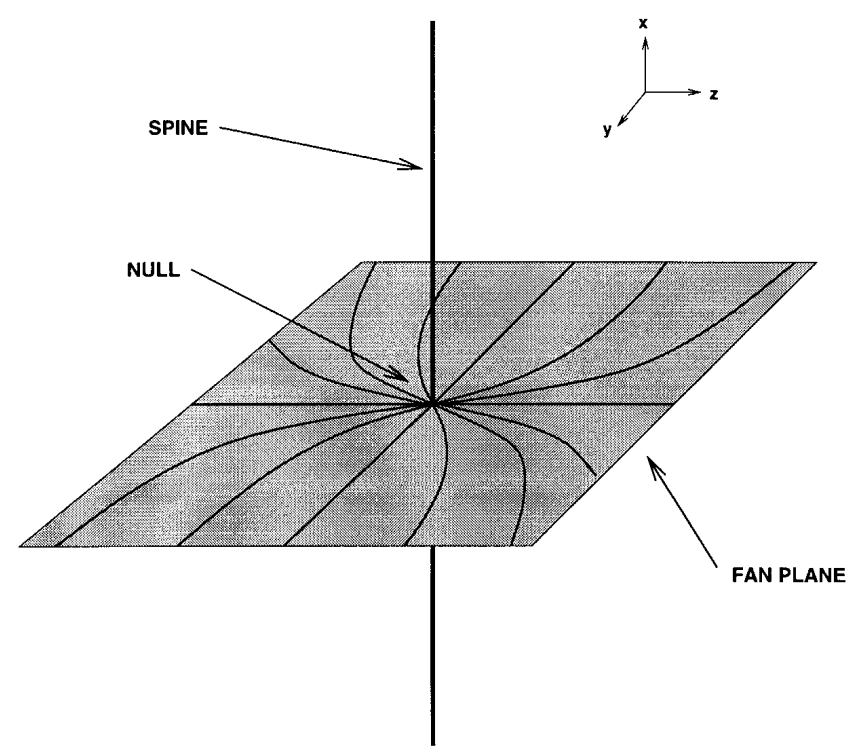

FIG. 1.-Schematic spine and fan structure for an isolated $X$-point null 

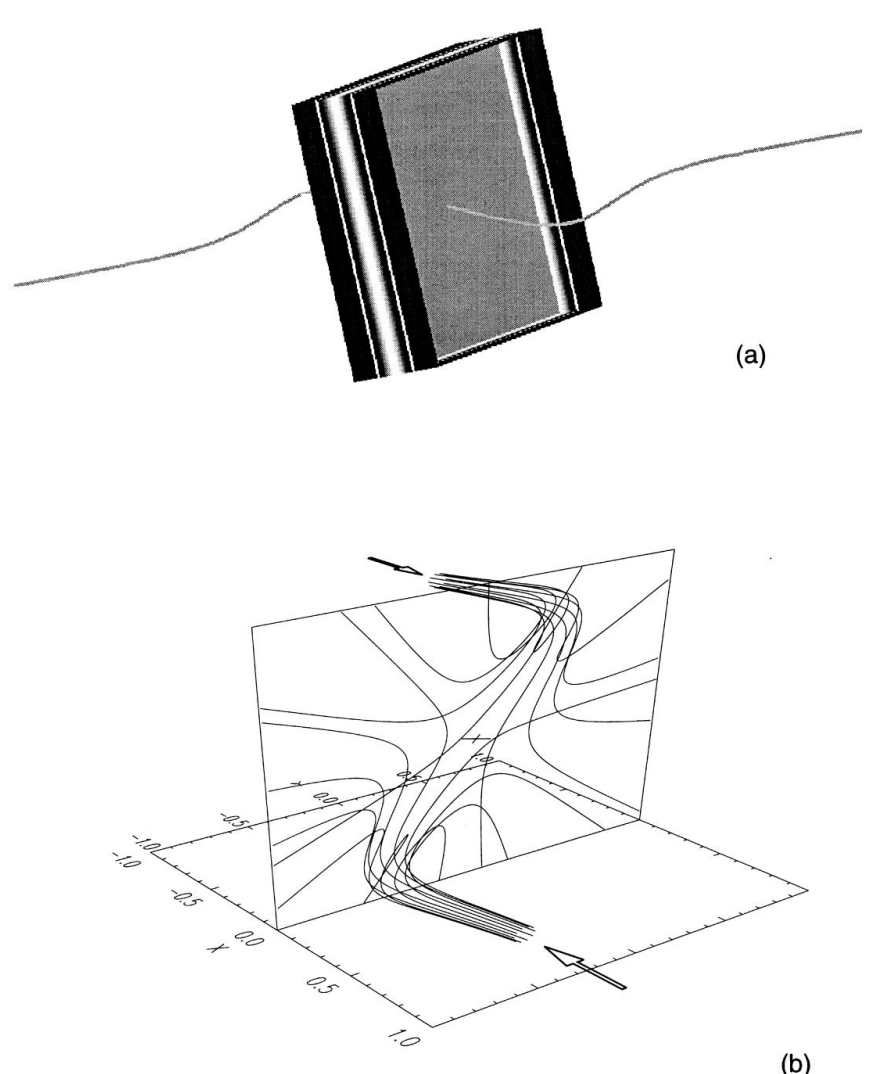

(b)

Fig. 2.-Fan reconnection solutions. (a) Schematic representation of the current sheet structure of a typical fan solution. Motions that distort the spine generate currents in the fan plane. Light shaded areas represent regions with high current magnitudes. (b) Flow streamlines for a fan reconnection solution with parameters $\kappa=\frac{1}{2}, \alpha=1, \lambda=\frac{3}{4}, \eta=3 \times 10^{-3}$, and $Y(1)=Z(1)=\frac{1}{4}$. The arrows denote the inflow direction, while the cross marks the position of the null.

spine solutions offer much richer current structures than the simple current sheets of the fan model. They also allow faster ohmic dissipation, provided the severe pressure problem discussed in $\S 3.2$ can be ignored.

\subsection{Fan Reconnection Scalings}

Let us first determine the dissipation scalings for fan current solutions. By substituting equation (2.6) into the momentum equation (2.1) and the induction equation (2.2) we find that the fan current disturbance field $Y(x)$ satisfies (Craig et al. 1995)

$$
\bar{\eta} Y^{\prime \prime}+x Y^{\prime}+\kappa Y=0, \quad \bar{\eta} \equiv \frac{\eta}{\alpha\left(1-\lambda^{2}\right)}, \quad \alpha>0,
$$

where $\bar{\eta}$ is of order $\eta$. An identical equation holds for $Z(x)$ under the replacements $Y(x) \rightarrow Z(x), \kappa \rightarrow(1-\kappa)$.

Instead of the boundary-layer analysis of Craig et al. (1995), we invoke here the parabolic cylinder function solution

$Y(x)=A \frac{x}{\bar{\eta}^{1 / 2}} M\left(\frac{\kappa+1}{2}, \frac{3}{2},-\frac{x^{2}}{2 \bar{\eta}}\right)+B M\left(\frac{\kappa}{2}, \frac{1}{2},-\frac{x^{2}}{2 \bar{\eta}}\right)$,

written in terms of the Kummer function $M(a, b, z)$. Wellbehaved solutions require $\bar{\eta}>0$, which implies either $\alpha>0$, $|\lambda|<1$ or $\alpha<0,|\lambda|>1$. We reject the latter possibility as unphysical since it implies small length scales on the inflow boundaries (Craig \& Henton 1995).
We require only the asymptotic properties of the Kummer function, namely,

$$
M(a, b, z) \sim \begin{cases}1+a z / b, & \text { as } z \rightarrow 0, \\ |z|^{-a}, & \text { as } z \rightarrow-\infty .\end{cases}
$$

We do not require the even solution (see Watson \& Craig 1997a, 1997b), so we set $B=0$. The odd solution grows linearly for small $x$, peaks when $x \simeq \eta^{1 / 2}$, and falls off as $A \eta^{\kappa / 2} x^{-\kappa}$ (recall we assume $\bar{\eta} \simeq \eta$ ). As $\kappa \rightarrow 1$ the flux pileup is most severe, and, for prescribed field strengths of order unity on $x= \pm 1$, the amplitude of the disturbance field scales as $A \sim \eta^{-\kappa / 2}$. By contrast, for $\kappa=0$ the field amplitude $Y(x)$ is constant outside the current layer. These extremes are simply the Dawson function and error function solutions, respectively, discussed by Fabling \& Craig (1966). Since our main concern is an estimate of the power output, we shall adopt only the simplest solution: this is the $\kappa=\frac{1}{2}$ fan solution in which the disturbance field $Q$ builds up as $\eta^{-1 / 4}$ at the onset of the current layer.

\subsection{Spine Reconnection Scalings}

Turning now to spine reconection, we note that $\kappa=\frac{1}{2}$ is again the simplest model, since it allows a cylindrical mode decomposition of the disturbance field in the planes of constant $x$. Specifically, we let

$$
X(y, z) \rightarrow X(r, \theta)=f(r) e^{i m \theta},
$$

where $r^{2}=y^{2}+z^{2}$ and $\tan \theta=y / z$. The radial spine equation for $f(r)$ is then given by

$$
f+\frac{1}{2} r f^{\prime}=\bar{\eta}\left(f^{\prime \prime}+\frac{f^{\prime}}{r}-\frac{m^{2}}{r^{2}} f\right) .
$$

For the solution to be well behaved we require $\bar{\eta}<0$ with $\alpha<0,|\lambda|<1$, which corresponds to plasma, injected into the fan, being squirted out along the spine. It is easily shown that only the $m=1$ mode (Fig. 3 ) corresponds to reconnection involving finite currents at the neutral point: all other modes are associated with pure resisitive diffusion in the cylindrical current layer (Craig \& Fabling 1996).

The solution, which is well behaved as $r \rightarrow 0$, is given by

$$
f(r)=A\left(\frac{r^{2}}{|4 \bar{\eta}|}\right)^{m / 2} M\left(1+\frac{1}{2} m, 1+m,-\frac{r^{2}}{|4 \bar{\eta}|}\right) .
$$

The $m=0$ mode is exceptional, but for all higher modes $f(r)$ falls off as $r^{-2}$ outside the axial region $r \sim \eta^{1 / 2}$ of high current density. We must therefore take $A \sim \eta^{-1}$ to achieve fields of order unity on the $r=1$ boundary. By using the Kummer transformation

$$
M\left(1+\frac{1}{2} m, 1+m,-z^{2}\right)=e^{-z^{2}} M\left(\frac{1}{2} m, 1+m, z^{2}\right),
$$

we can show that the Kummer function solutions, for all even $m$, reduce to the terminating series solutions deduced by Craig \& Fabling (1996). More critically, as discussed below, the field amplitude $|f(r)| \simeq A \sim \eta^{-1}$ leads to a superfast dissipation rate.

\section{POWER OUTPUT FOR SPINE AND FAN RECONNECTION}

\subsection{Introduction}

The solutions outlined above offer an exact description of steady state incompressible reconnection in open geome- 


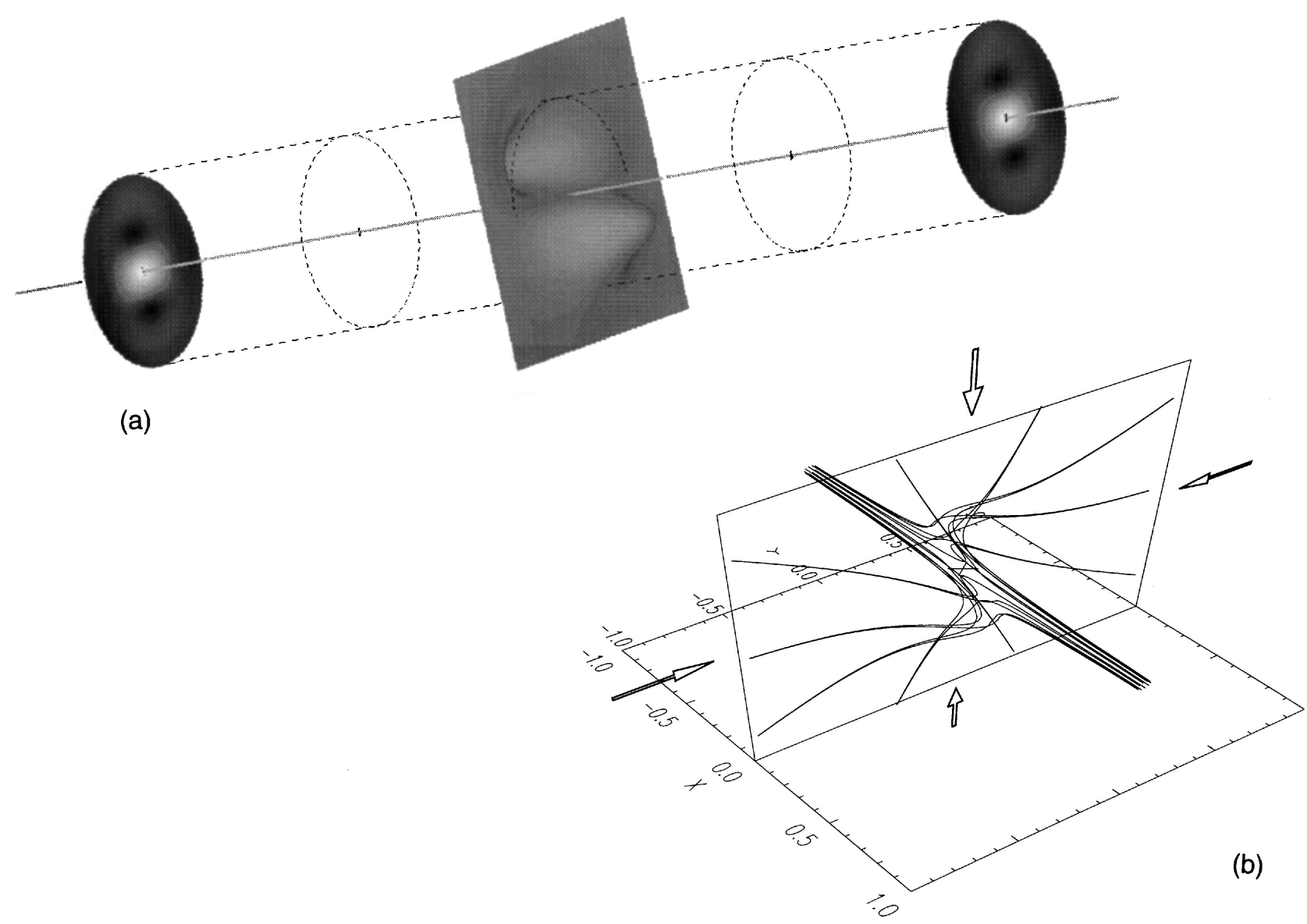

FIG. 3.-Spine reconnection solutions. (a) Schematic representation of the current structure of a typical spine solution. Motions that distort the fan generate currents along the spine. In contrast to the sheet geometry of Fig. 1, the currents are now constrained to quasi-cylindrical tubes. $(b)$ Flow streamlines for spine reconnection. Inflow aligned to the fan is squired out along the spine. Parameters are $\kappa=\frac{1}{2}, \alpha=-1, \lambda=\frac{3}{4}, \eta=3 \times 10^{-3}, m=1$, and $f(1)=0.015$.

tries. However, as we will see in $\S 3.2$, fast reconnection is achieved at the high price of unrealistic hydromagnetic pressures throughout the reconnection region. This leads to the absurd result that, for fixed-amplitude disturbance fields on the inflow boundaries, the ohmic dissipation rate can increase indefinitely (see [3.3]) with reductions in the plasma resistivity.

To overcome this lack of physicality we need to impose an upper limit to the hydromagnetic pressures that can be tolerated in the reconnection region (Watson \& Craig 1997a, 1997b). The disturbance field must be limited to some saturated amplitude determined, for a prescribed hydromagnetic pressure bound, by the plasma resistivity $\eta$. We will see that this prescription leads to concrete predictions for the maximum power output at realistic coronal resistivities.

\subsection{The Problem with Fast Reconnection}

To determine the scaling of the dissipation rate, we must fix the level of the disturbance field $Q$ on the inflow boundaries of the model. Consider first the fan reconnection scalings for $\kappa=\frac{1}{2}$. The volume containing the current $\Delta V$ is proportional to the width $\Delta x \sim \eta^{1 / 2}$ of the sheet. If fields of order unity are washed in through the inflow boundaries $x= \pm 1$, the field amplitude at the current layer must scale as $|Q(x)| \sim \eta^{-1 / 4}$, and so

$$
W_{\eta} \simeq \eta J^{2} \Delta V \simeq Q^{2} \Delta V \sim \eta^{0} .
$$

This confirms $W_{\eta}$ as fast when fields of fixed amplitude are washed into the reconnection region.

The plasma pressure is obtained from the uncurled form of the momentum equation (2.1). The result is

$$
p(x, y, z)=p_{0}-\left(P^{2}+Q^{2}\right) / 2-\alpha \lambda[y Y(x)+z Z(x)] / 2,
$$

and so the pressure $p_{0}$ at the neutral point must increase without bound as $\eta \rightarrow 0$ in order for the pressure to remain positive. In particular, $p_{0} \simeq Q^{2} \sim \eta^{-1 / 2}$. This huge pressure reflects the magnetic pressure in the sheet and the hydromagnetic pressure on the inflow boundary $x=1$. It follows that pressure in the reconnection region is ultimately set (as a boundary condition for any fixed $\eta$ ) by the nature of the external regions that drive the reconnection. Equation (3.2) then confirms that the external hydromagnetic pressures (at $x= \pm 1$ ) required to sustain the reconnection must be comparable to the gas pressure $p_{0}$ at the neutral point.

The pressure problem is even more severe for spine reconnection. The disturbance field builds up as $Q=X \simeq \eta^{-1}$, and since $\Delta V \simeq \pi r^{2} \sim \eta$ we obtain the superfast rate

$$
W_{\eta} \simeq \eta J^{2} \Delta V \simeq Q^{2} \Delta V \sim \eta^{-1} .
$$

Instead of equation (3.2) we now have

$$
p(x, y, z)=p_{0}-\left(P^{2}+X^{2}\right) / 2+\alpha \lambda x X,
$$

and the scaling of the pressure, namely, $p_{0} \simeq X(y, z)^{2} \sim \eta^{-2}$, is even more extreme. 


\subsection{Saturated Power Output for Fan Reconnection}

We now limit the output power by assuming that the hydromagnetic pressure of the neutral-point region is bounded by the external pressure available to drive the merging. Since the largest pressures in the solar atmosphere are associated with photospheric sunspot fields of around $B_{p}=10^{3.5} \mathrm{G}$, we assume that the localized magnetic field surrounding the neutral point can be enhanced (relative to our reference coronal field $B_{c}=100 \mathrm{G}$ ) by factors not exceeding $10^{1.5}$. Thus $B_{p} \simeq 10^{1.5}$ in the nondimensional units of $\S 2.2$.

In the case of fan reconnection we assume the limit $|Q(x)| \simeq B_{p}$ for $\eta \simeq \eta_{c}$. This gives the saturated output

$$
W_{\eta_{c}} \simeq B_{p}^{2} \Delta V \simeq \eta_{c}^{1 / 2} B_{p}^{2} \simeq 10^{-3},
$$

assuming $\eta_{c}=10^{-12}$. Since our unit of power is $10^{30} \mathrm{ergs}$ $\mathrm{s}^{-1}$, we achieve our target rate of $10^{27} \mathrm{ergs} \mathrm{s}^{-1}$.

How much energy is actually liberated during the reconnection process? If we evaluate the total energy in the disturbance field, we find that [by a factor of $\left.\ln \left(1 / \eta_{c}\right)\right]$ the outer region $x>\eta^{1 / 2}$ accounts for the bulk of the excess magnetic energy,

$$
\left\langle\frac{1}{2} B^{2}\right\rangle \simeq \frac{1}{4} \eta_{c}^{1 / 2} B_{p}^{2} \ln \left(\frac{1}{\eta_{c}}\right) \simeq 10^{-2} .
$$

This translates to liberating $2 \times 10^{29}$ ergs in the disturbance field in typically

$$
\tau \simeq \frac{\left\langle B^{2} / 2\right\rangle}{W_{\eta_{c}}}=\frac{1}{4} \ln \left(\frac{1}{\eta_{c}}\right) \simeq 10
$$

coronal Alfvén times. This corresponds to a time of around $10^{2}$ s typically.

\subsection{Saturated Power Output for Spine Reconnection}

For spine reconnection we assume the bound $|X(y, z)| \equiv$ $|f(r)| \simeq B_{p}$ when $\eta \simeq \eta_{c}$. However, because of the small volume element $\Delta V \simeq \pi r^{2} \sim \eta$, we obtain a saturated output of only

$$
W_{\eta_{c}} \simeq B_{p}^{2} \Delta V \simeq \pi \eta_{c} B_{p}^{2} \simeq 10^{-8.5} .
$$

This is down by a factor of $10^{5.5}$ on our target rate of $10^{27}$ ergs $\mathrm{s}^{-1}$ and suggests that spine reconnection is incapable of powering even a tiny flare. The problem lies in the geometry: we find that the energy contained in the field,

$$
\left\langle B^{2} / 2\right\rangle \simeq \pi \eta_{c} B_{p}^{2} \simeq 10^{-8.5},
$$

translates to only $6 \times 10^{22}$ ergs. Thus, although the energy is released over a single Alfvén time, that is,

$$
\tau \simeq \frac{\left\langle B^{2} / 2\right\rangle}{W_{\eta_{c}}} \simeq 1,
$$

the quasi-cylindrical spine region simply cannot contain enough magnetic energy under the constraint that $|f(r)| \leq$ $B_{p}$ for $\eta \simeq \eta_{c}$. Obviously, the condition of fast dissipation is only necessary, not sufficient, for a significant power output.

\subsection{Geometrical Considerations}

It is interesting that the previous results can be anticipated on very simple geometrical considerations, independent of the details of the spine and fan solutions. Suppose $\Delta V$ is the current volume enclosing the flux pileup field of amplitude $Q$. We shall assume, in line with the previous solutions, that $\Delta V$ contains a representative fraction of the magnetic energy in the disturbance field $\boldsymbol{Q}$. Suppose further that

$$
\Delta V \simeq l^{v} L^{3-v}, \quad L \sim \eta^{0},
$$

where $l$ scales in some way with $\eta$, and $v \geq 1$ governs the dimensionality of the current region. Then $v=1$ gives a current sheet, appropriate to fan current reconnection, while $v=2$ represents the current cylinder of spine reconnection. The case $v=3$ has no direct interpretation in terms of the present solutions but can be thought of as a localized current ball. In the same sense $v=1.5$ (for example) corresponds to a flattened cylinder of current.

Since $J \sim Q / l$, the power and energy scalings are given by

$$
\begin{aligned}
& W_{\eta} \simeq \eta Q^{2} l^{v-2} L^{3-v}, \\
& \left\langle B^{2} / 2\right\rangle \simeq Q^{2} l^{v} L^{3-v} .
\end{aligned}
$$

Obviously we would like both these quantities to scale favorably with $\eta$. While $Q$ should be chosen as large as possible (consistent with $Q^{2} \leq B_{p}^{2}$ ), the scaling with $l$ is contradictory. However, by taking $l \sim \eta^{1 / 2}$, we obtain identical scalings for both $W_{\eta}$ and $\left\langle B^{2} / 2\right\rangle$ for any dimensionality $v$. This corresponds to the invariant dissipation timescale

$$
\tau \simeq \frac{\left\langle B^{2} / 2\right\rangle}{W_{\eta}} \simeq \frac{l^{2}}{\eta} \sim \eta^{0} .
$$

It seems a remarkable coincidence that both the spine and fan solutions possess this $\eta^{1 / 2}$ scaling. It is clear that in this case the dimensionality $v=1$, corresponding to the fan current solution (eq. [3.5]), maximizes the amount of energy that can be released.

These arguments, of course, are not restricted to the spine and fan solutions: they apply equally well to timedependent reconnection models. Consider, for instance, the case of planar reconnection driven by the coalescence instability, as in Biskamp \& Welter (1980) and Rickard \& Craig (1993). Here the sheet is quasi-one-dimensional $(v=1)$ with only the width dependent on $\eta$. In the flux pileup regime the instability drives the sheet according to $l \sim \eta^{2 / 3}, Q \sim \eta^{1 / 3}$. Thus a superfast dissipation rate $W_{\eta} \sim \eta^{-1 / 3}$ is obtained at the expense of the energy in the sheet $\left\langle B^{2} / 2\right\rangle \sim \eta^{0}$. By contrast, in the planar reconnection experiment of Deluca \& Craig (1992), $v=1, l \sim \eta^{1 / 2}, Q \sim \eta^{-1 / 4}$, and so an invariant dissipation timescale is maintained without compromising the fast dissipation rate $W_{\eta} \sim\left\langle B^{2} / 2\right\rangle \sim \eta^{0}$.

Of course, in any concrete case, the reconnection rate is determined by the details of the simulation, which involves both the magnetic geometry and the boundary and initial conditions of the problem. Watson \& Craig (1997a, 1997b) have already demonstrated that multiple scalings can arise in magnetic geometries which comprise several nulls. Although such models offer intriguing possibilities, the present argument suggests that of all the possible flux pileup models, the scaling of the three-dimensional fan solution offers the best compromise.

\subsection{Physical Interpretation}

The previous results suggest that fan current reconnection is most likely to meet the energetic constraints of the flare. Even so, the largest flares seem well beyond the saturated fan output based on the classical resistivity $\eta=\eta_{c}=$ $10^{-12}$. This difficulty is eased considerably, however, if we make the plausible assumption that microinstabilities enhance the resistivity by some typical factor $F$. In fact, since the physical extent of the high current region is below the collisional mean free path of the plasma, some break- 
down of the classical resistivity is inevitable. Since estimates (e.g., Parker 1979) suggest $F \simeq 10^{4}$, both the fan output power and the magnitude of the energy release can be raised by the amount $F^{1 / 2}=10^{2}$, without altering the dissipation timescale (eqs [3.5], [3.6], and [3.7] above). Alternatively, for a fixed saturated output, the hydromagnetic pressure in the fan reconnection region could be eased by an order of magnitude $F^{-1 / 4}$, to a level which barely exceeds the typical coronal value.

We recognize, of course, that there are many other possibilities for enhancing the classical dissipation rate. Aside from other mechanisms for enhancing the resistivity (e.g., by turbulent convection), we have not considered the influence of time-dependent effects (e.g., Politano, Pouquet, \& Sulem 1995; Craig \& McClymont 1997), or the finite compressibility of the plasma (e.g., Scheper \& Hassam 1995; Rickard \& Titov 1996). Multiple null solutions also appear promising at first sight. But although these can involve multiple length scales and allow several sites for the energy dissipation, it seems difficult to achieve much improvement over the present fan reconnection scalings (Watson \& Craig 1997a, 1997b).

Turning to time-dependent effects, we note that the main feature of the saturated power calculation is the need to limit the amplitude of the disturbance field on the inflow boundary. This field is strongly amplified by the inflow-by factors of 100 or more - and eventually piles up in a narrow current layer overlying the neutral point. It is the back pressure generated in the flux pileup layer that stalls the reconnection and leads to saturation. It is possible to imagine situations, however, where the outer field region becomes causally decoupled from the neutral point-for example, in dynamic compressible plasmas where nearvacuum regions develop at the onset of the sheet. Such extreme situations may well allow a relaxation of the steady state saturation limit.

\section{CONCLUSIONS}

We have considered the energy output characteristics of three-dimensional spine and fan magnetic reconnection solutions. Although these models have the potential for rapid energy release, the ohmic dissipation rate is fast only if the hydromagnetic pressure in the reconnection region builds up continually with reductions in the plasma resistivity, $\eta$. We have argued, following Watson \& Craig (1997a, 1997b), that the important energetic quantity in this case is the saturated power output obtained by limiting the hydromagnetic pressure in the model to levels available in the solar atmosphere. This automatically overcomes the physical implausibility of accelerating dissipation rates as $\eta \rightarrow 0$. It is also consistent with dynamic merging simulations which show that the reconnection rate stalls when the back pressure in the current layer overcomes the hydromagnetic (boundary) forces driving the reconnection (see Biskamp 1994; Craig et al. 1993).

Of the two reconnection solutions considered, it seems the fan model is the more promising flare candidate. The narrow cylindrical current structures $r \sim \eta^{1 / 2}$ of the spine solution contain insufficient magnetic energy for the flare, at least within the limits imposed by the saturated field amplitudes. By contrast, the current sheet of the fan solution is energetically far more favorable: our estimates suggest that it can account for a modest flare even without invoking the likely enhancement of the classical resistivity by microinstabilities.

In the present study we have considered only the simplest, exact three-dimensional reconnection solutions, yet we know from Watson \& Craig (1997a, 1997b) that other reconnection solutions can be constructed, possibly with more favorable dissipation scalings. The geometrical arguments of $\S 3.5$ suggest, however, that unless the scaling $l \sim \eta^{1 / 2}$ is obtained, either the power output or energy release will be adversely affected. In meeting this requirement, we believe the current generation of exact, threedimensional reconnection solutions offer encouraging possibilities for interpreting the flare release.

We thank Ernie Kalnins for encouraging us to express our solutions in terms of named functions. P. G. W. acknowledges the support of NZ FRST funding.
Biskamp, D. 1994, Phys. Rep., 237, 179

Biskamp, D., \& Welter, H. 1980, Phys. Rev. Lett., 44, 1069

Craig, I. J. D. 1994, A\&A, 283, 331

Craig, I. J. D., \& Fabling, R. B. 1996, ApJ, 462, 969

Craig, I. J. D., Fabling, R. B., Henton, S. M., \& Rickard, G. J. 1995, ApJ, 455, L197

Craig, I. J. D., \& Henton, S. M. 1995, ApJ, 450, 280

Craig, I. J. D., Henton, S. M. \& Rickard, G. J. 1993, A\&A, 267, L39

Craig, I. J. D., \& McClymont, A. N. 1997, ApJ, 481, 996

Craig, I. J. D., \& Watson, P. G. 1992, ApJ, 393, 385

DeLuca, E. E., \& Craig, I. J. D. 1992, ApJ, 390, 679

Fabling, R. B., \& Craig, I. J. D. 1996, Phys. Plasmas, 3, 2243

Forbes, T. G., \& Priest, E. R. 1987, Rev. Geophys., 25, 1583

Hassam, A. B. 1992, ApJ, 399, 159

\section{REFERENCES}

Lau, Y. T., \& Finn, J. M. 1990, ApJ, 350, 672

McClymont, A. N., \& Craig, I. J. D. 1996, ApJ, 466, 487

Ofman, L., Morrison, P. J., \& Steinolfson, R. S. 1993, ApJ, 417, 748

Parker, E. N. 1979, Cosmical Magnetic Fields (Oxford: Clarendon)

Politano, H., Pouquet, A., \& Sulem, P. L. 1995, Phys. Plasmas, 2, 2931

Priest, E. R., \& Titov, V. S. 1996, Philos. Trans. R. Soc. London, A, 354, 2951

Rickard, G. J., \& Craig, I. J. D. 1993, Phys. Fluids, B, 5, 956

Rickard, G. J., \& Priest, E. R. 1994, Sol. Phys., 151, 107

Rickard, G. J., \& Titov, V. S. 1996, ApJ, 472, 840

Scheper, R. A., \& Hassam, A. B. 1995, ApJ, 455, 693

Watson, P. G., \& Craig, I. J. D. 1997a, Phys. Plasmas, 4, 101

—. 1997b, Phys. Plasmas, 4, 110 